\title{
An In-Vitro Evaluation of Remaining Dentine Thickness through Cbct Using Different Files
}

\author{
Anil K Tomer ${ }^{1}$, Anjali Miglani ${ }^{2}$, Priyali Chauhan ${ }^{3}$, P. Nagarjuna ${ }^{4}$, \\ Sandeep Rana ${ }^{5}$, Anamika Kumari ${ }^{6}$ \\ ${ }^{I}$ MDS, Professor And Head, Department Of Conservative Dentistry \& Endodontics, D.J College Of Dental \\ Sciences And Research, Modinagar. \\ ${ }^{2}$ MDS, Reader, Department Of Conservative Dentistry \& Endodontics, D.J College Of Dental Sciences And \\ Research, Modinagar. \\ ${ }^{3,4,5,6}$ Post Graduate Student, Department Of Conservative Dentistry \& Endodontics, D.J College Of Dental \\ Sciences And Research, Modinagar.
}

\begin{abstract}
Aim: The study was to evaluate the effect of multiple file system (Revo S) versus single file system (one shape) on the dentine thickness in the root canal.

Materials and Methods: In this study, 30 freshly extracted human mandibular first molars were taken. Mesiobuccal canal of mandibular first molar was selected for this study. All the teeth were categorized into two groups namely Group I: Revo -S Group II: One Shape. Endodontic access was prepared in each tooth, and working length was determined using 15-k file. Pre-operative cone beam computed tomography (CBCT) scan of the teeth was taken. Cleaning and shaping of the canal was done according to the manufacturer's instruction of file system. Post instrumentation CBCT scan of teeth was taken. The difference in the area was calculated and statistical difference was analyzed.

Result: One shape removed less dentin than Revo-S file system. Conclusion: Revo- S and One Shape showed statistically signifi cant difference in remaining dentin thickness over the different intervals of root canal length. Therefore, it was concluded that One Shape file system removed less dentine than Revo-S file system
\end{abstract}

\section{Introduction}

Root canal preparation has been considered the most important step in endodontic therapy for dentin removal $^{1}$. Residual dentin thickness indicates the mechanical limits of instruments, to enlarge the diameter of the root canal, to approximately predetermined values that would not significantly weaken the dentinal walls ${ }^{2}$. Strip perforation and vertical root fractures are possible outcomes of excessive removal of radicular dentin especially in zones that have been termed "danger zones"3.

Cleaning the root canal system of organic remnants and removing debris and microorganisms from the apical portion of the root canal are important steps in successful endodontic treatment ${ }^{4}$. Nickel-Titanium (NiTi) alloy instruments have recently become an integral part of root canal treatment. Numerous studies have shown that Ni-Ti rotary instruments can effectively produce a well-tapered root canal form sufficient for filling with minimal risk of transportation..These instruments are two to three times more flexible than stainless steel files and superior to them in terms of angular deflection and maximum torque to failure ${ }^{5}$. The evaluation of root canal instrumentation is necessary to determine the action of the instruments on the original canal shape and to observe if the principles of canal preparation had been followed. СBCT evaluation has shown that the exact location and anatomy of the root canal system can be assessed and it has been validated as a tool to explore root canal anatomy ${ }^{6}$.

\section{Material and methods}

Forty extracted human mandibular molars were taken. Each tooth was sectioned through the furcation and mesiobuccal canal was used. Conventional endodontic access was done on each tooth. Working length was determined using a size 15-k (Dentsply Mailleff er) fi le.

- All the teeth were allocated into two groups with 20 teeth in each :

Group I: REVO S ( Micro mega)

Group II: One Shape (Micro-Mega)

Pre-instrumentation Axial section measuring the diameter of the root canal and the amount of dentin remaining both on right and left side of the canal using C S 3D Software at 7mm. ( figure 1) 


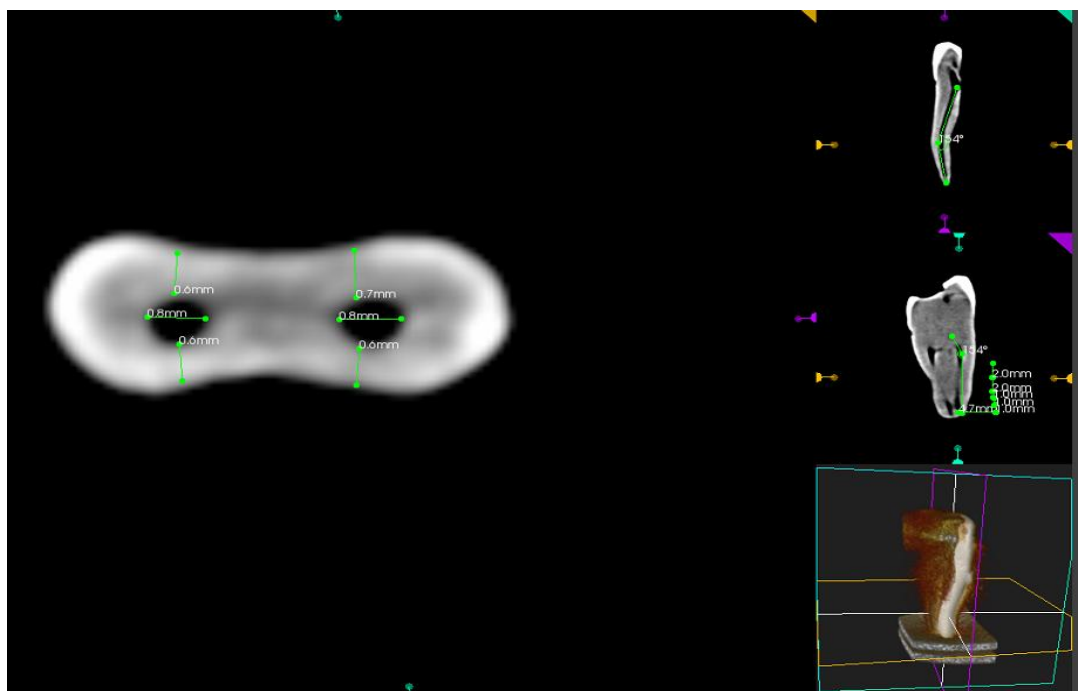

Figure 1 Pre instrumentation axial section

Cleaning and shaping of the canal was done according to the group assigned following manufacture's instructions. Post-instrumentation Axial section measuring the diameter of the root canal and the amount of dentin remaining both on right and left side of the canal using C S 3D Software at $7 \mathrm{~mm}$ ( Figure 2) The pre and post instrumentation images of OneShape were superimposed using ON DEMAND 3D FUSION software at $7 \mathrm{~mm}$ (Figure 3)

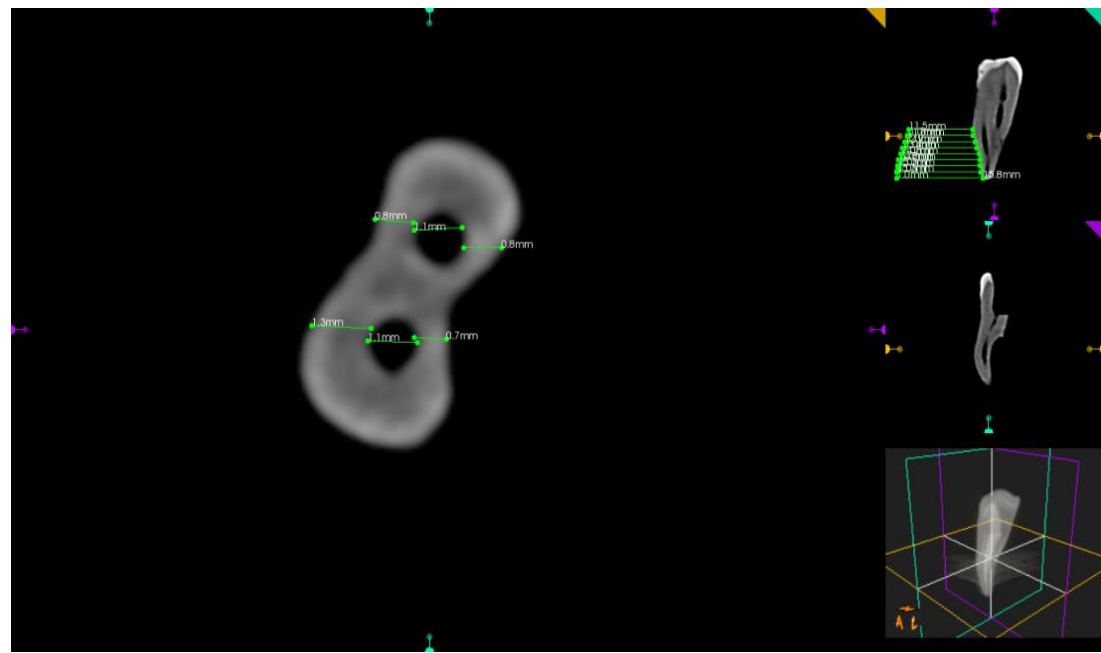

Figure 2 Post instrumentation axial section

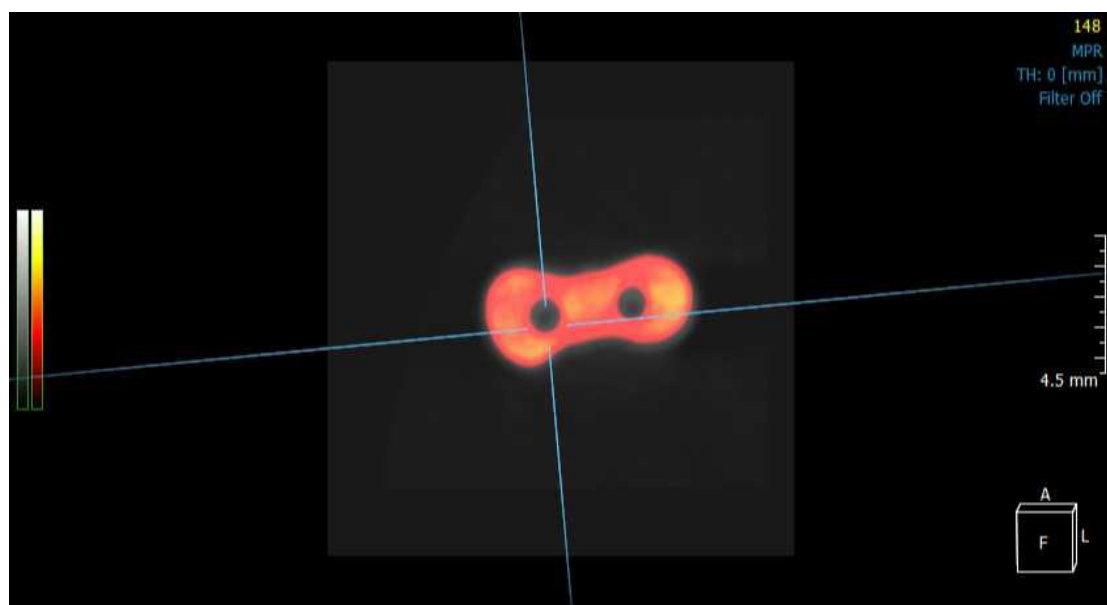

Figure 3 Superimposition of images 


\section{Results}

The descriptive statistics of remaining dentin thickness for all the two file systems at $0 \mathrm{~mm}, 1 \mathrm{~mm}, 2 \mathrm{~mm}$, $3 \mathrm{~mm}, 4 \mathrm{~mm}, 5 \mathrm{~mm}, 6 \mathrm{~mm}, 7 \mathrm{~mm}$ were calculated. Tables 1 and 2 show the descriptive statistics of remaining dentin thickness in all the two fi le systems.

Table 1: Independent $t$-test used to calculate the $P$ value at diff erent intervals of root length among various fi le systems $(P<0.05)$

Groups*

I-II

$$
P \text { value }
$$

$0 \mathrm{~mm} \quad 1 \mathrm{~mm} 2 \mathrm{~mm} \quad 3 \mathrm{~mm} 4 \mathrm{~mm} 5 \mathrm{~mm} 6 \mathrm{~mm} 7 \mathrm{~mm}$ $\begin{array}{llllllll}0.020 & 0.088 & 0.022 & 0.014 & 0.006 & 0.093 & 0.017 & 0.020\end{array}$

*Equal variances assumed. *Groups I: Revo S , II: One shape

Mean value and standard deviation of dentin removal is maximum at all the levels for Revo $s$ than One Shape.

Table 2: Descriptive statistics to calculate remaining dentine thickness using one-way ANOVA test

\begin{tabular}{|lllccc|}
\hline Intervals & $n$ & One shape(M) & Revo S $(M)$ & One shape (SD $)$ & Revo S (SD) \\
\hline $0 \mathrm{~mm}$ & 40 & 0.028 & 0.0376 & 0.0864696479 & 0.0299215641 \\
\hline $1 \mathrm{~mm}$ & 40 & 0.0096 & 0.0414 & 0.2653588137 & 0.0298964881 \\
\hline $2 \mathrm{~mm}$ & 40 & 0.022 & 0.058 & 0.0274590604 & 0.3998208349 \\
\hline $3 \mathrm{~mm}$ & 40 & 0.0368 & 0.008 & 0.0376390754 & 0.2367667629 \\
\hline $4 \mathrm{~mm}$ & 40 & 0.0432 & 0.16 & 0.0343394817 & 0.5103423361 \\
\hline $5 \mathrm{~mm}$ & 40 & 0.0318 & 0.25 & 0.0143944434 & 0.3722479013 \\
\hline $6 \mathrm{~mm}$ & 40 & 0.044 & 0.088 & 0.0351468349 & 0.1118087653 \\
\hline $7 \mathrm{~mm}$ & 40 & 0.0514 & 0.2262 & 0.0310048383 & 0.1555480633 \\
\hline
\end{tabular}

SD: Standard deviation M: Mean

Signifi cant diff erence was found at $0 \mathrm{~mm}, 2 \mathrm{~mm}, 3 \mathrm{~mm}, 6 \mathrm{~mm}, 7 \mathrm{~mm}$ between the two fi le systems [Graph 1].

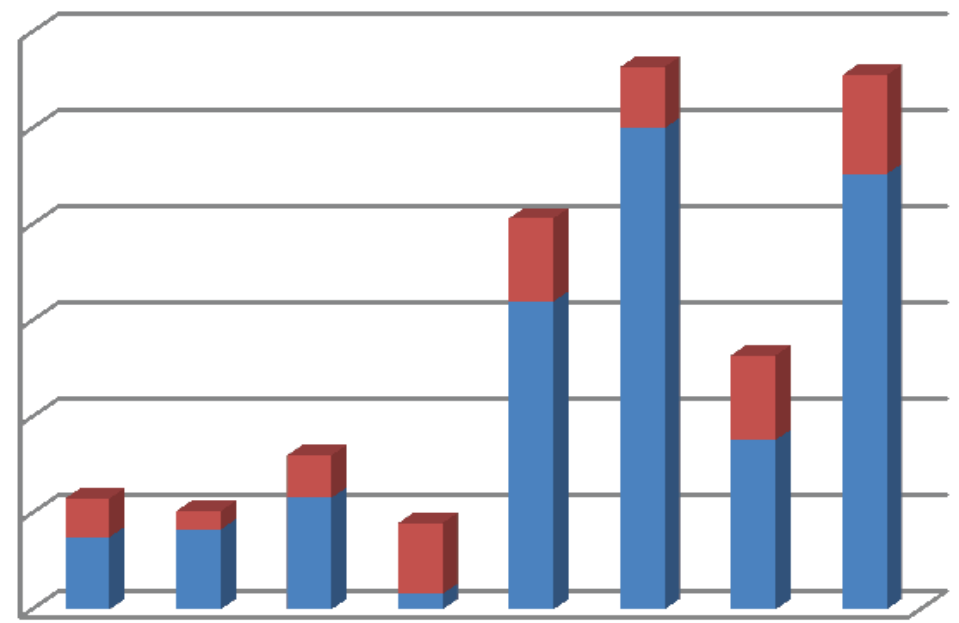

Graph 1: Calculation of remaining dentin thickness by different file systems at various intervals

\section{Discussion}

The variation of root dentine thickness in different areas supports the notion that it is very important for practitioners to increase their knowledge in regard to root canal anatomy ${ }^{3}$. The thickness of the dentine wall is directly proportional to the ability of the tooth to withstand lateral forces. Therefore, treatment that causes indiscriminate removal of tooth structure from the canal walls during endodontic treatment should be avoided ${ }^{7}$. Primary objectives of canal preparation include the removal of organic substrate from the canal system by chemo mechanical methods and the three dimensional shaping of the root canal system into a continuously tapering preparation while maintaining the original outline form of the $\mathrm{canal}^{8}$. These objectives are often difficult to achieve because of variations in canal cross sectional shape, presence of anatomical irregularities and canal curvature, which often occurs in more than one direction. Stainless steel instruments possess reduced 
flexibility in larger sizes and tend to straighten in curved canals. This results in an increased incidence of procedural errors such as ledge, zip, elbow formation, canal transportation and stripping 9 .

The introduction of nitinol to endodontics provided a material with superior flexibility and resistance to torsional fracture. These properties enabled improved design features, which reduce procedural errors and result in canal preparations that maintain the original canal form when either rotary or hand instrumentation is employed $^{10}$.

Revo-STM, a unique and innovating sequence. Intended for initial endodontic treatments, Revo-S ${ }^{\mathrm{TM}}$ innovates with only 3 instruments. Its asymmetrical section initiates a snake-like movement of the instrument inside the canal. The smaller section allows more flexibility and offers a better ability to negotiate curves. The asymmetrical cross-section increases the available volume for upward debris elimination. The instrument works in a cyclic way (3C Concept): 1) Cutting 2) Clearance (debris elimination) 3) Cleaning ${ }^{11}$.

One shape single file system provides simplicity to practitioners: Simplicity of use: single instrument for canal shaping, Flexibility: superior ability to negotiate curves ,Efficiency: in continuous rotation , Safety: single use , Innovation: new asymmetrical cross-section with longer pitch. CBCT allows evaluating root canal preparation without cutting off the specimens and loosing the root canal structure ${ }^{12}$.

\section{Conclusion}

Excessive removal of radicular dentin compromises the root. A direct relationship exists between the RDT to the strength of the root .Thus, preservation of sound dentin is of utmost importance. At least $1 \mathrm{~mm}$ of root dentin should remain in all root aspects along its entire length after all intra-radicular procedures are completed $^{13}$. The distolingual aspect of the root canal is a danger zone for the mesiobuccal canal of the mesial root in mandibular molar teeth, and preparation of that area should be performed with caution ${ }^{5}$. 